\title{
A random graph model for optical networks of sensors*
}

\author{
Josep Díaz Jordi Petit Maria Serna \\ Departament de Llenguatges i Sistemes Informàtics \\ Universitat Politècnica de Catalunya \\ Jordi Girona Salgado 1-3 \\ 08034 Barcelona \\ \{diaz,jpetit,mjserna\}@|si.upc.es
}

\begin{abstract}
In this paper we propose a model for Smart Dust Networks and show its applicability when the goal of the network is monitoring an area under the surveillance of a base station. We analyze basic parameters of these nets and simple distributed protocols. These protocols provide a communication layer for networks of sensors that communicate through optical links.
\end{abstract}

\section{Introduction}

The Smart Dust project at UC Berkeley is a promising approach to build wireless ad-hoc network of very small sensors [KKRP99, Sma, KKP00]. As opposed to most other networks of sensors, these smart dust systems use optical communication rather than radio frequency. The current advances in hardware technology, digital circuitry, wireless communication and micro electromechanical systems allow the development of such networks. However, as reported in [KKRP99]: The missing ingredient is the networking and application layers needed to harness this revolutionary capability into a complete system. In this paper, we propose a random model to analyze the performance of smart dust networks communicating through optical devices. We also consider and analyze some basic algorithms for communication in the proposed model. Such algorithms represent a current research challenge, mainly because of the optical nature of these networks, and the presence of faulty connections among sensors.

One of the settings of smart dust systems is to have a base-station transceiver (BTS) at a relative elevation (or in a small plane) monitoring periodically the information of a large amount of sensors (motes) that have been scattered on a terrain. Smart dust systems are supposed to be deployed in hostile environments, in order to permit monitoring and tracking of remote objects, detecting anomalous situations as fires in the woods, movement of faults, etc [KKP00, EGHK99, Sma, ASSC02].

Motes are designed to be small, a few cubic millimeters, light and cheap. Besides sensing devices, motes are equipped with a tiny battery, a solar cell, a simple processor, a clock, a small memory and communication devices. In order to minimize size and energy, the last generation of motes use free-space optical transmission rather than radio frequency. In this case, motes include a small laser cannon and a set of optical devices able to modulate the

${ }^{*}$ Work partially supported by the IST Programme of the EU under contract number IST-2001-33116 (FLAGS) and by the Spanish CICYT project TIC-2001-4917-E. 
light they receive [KKRP99]. Free-space optical links have the limitation of an uninterrupted line-of-sight path for communication, but avoid radio interference. Current technology makes possible to store in a mote a total amount of energy on the order of 1 Joule, which implies that the power consumption of sensors is limited to microwats level. As a consequence, power management becomes a main ingredient in the design of algorithms.

In general, the communication will be initiated from the BTS: the BTS scans an area with its laser, and each mote passively modulates and reflects the beam. This is the preferred way to communicate, as the mote spends little power. The BTS can successfully decode simultaneous transmissions of dust motes, provided that the motes do not block one to another's line of sight to the BTS, which in view of the motes' small size and the relative elevation of the BTS, can be considered to be unlikely; however, as the motes are scattered massively at random from a vehicle, some of them may fall in such a way that can not communicate with the BTS. If a mote is shadowed from the BTS, this mote must rely its information to the BTS through other motes. Any mote can detect a failure of communication with the BTs by noticing that sufficient time has passed without communication. Communication between motes is done in an active way using their laser beams. This kind of transmission uses more power. To send information, they use an orientable low power laser beam, which current technology allows to move sidewards and upward about forty degrees [Pis02]. To receive information, motes have an optical device able to detect and interpret laser signals, as well as to evaluate the direction of the incoming beam.

In this paper, we consider two main phases before such a network can become operative. The first phase consists in finding out the position of each mote. To process sensor data, it is imperative to know where the data is coming from. At the end of this phase, it is required that all operative motes know their approximate coordinates. Such phase is called localization. GPS systems cannot be used because of their volume, price and power consumption. The second phase consists in establishing a route from the motes that cannot communicate with the BTS to the motes than can. Such phase is called route establishment. Once these two main steps are performed, the network is able to enter in its exploitation phase, which will last until the power supply of the motes decays.

The algorithms we present are analyzed on the proposed model for static smart dust networks. In our model, immobile motes are scattered at random in a two dimensional square terrain, taking into consideration that some of the motes become inoperative because they fall upside down, and that their laser beam can be oriented in any position of a prescribed scanning area, allowing for random failures in the mote-to-mote or mote-to-BTS communication.

We will deal with an ideal two dimensional squared region in which a mote falls with coordinates following the uniform distribution. The motes can orient its laser cannon in any position of a scanning area. This scanning area covers a (contiguous) sector of $\alpha$ degrees. The sector is also oriented randomly. We also introduce a new model of directed random geometric graphs that we call random scaled sector graphs. To the best of our knowledge this is the first time that such a model has been considered, and it might be of use to model other settings. When $\alpha=2 \pi$, random scaled sector graphs correspond to an directed version of random geometric graphs [AR97a, AR97b, DPS98].

All through the paper, we use standard terminology for directed and undirected graphs and for probability (see, for example, [MR95]). The distance $d(x, y)$ between two points in the plane will be the Euclidean distance. Recall that a sequence of events holds with high probability (w.h.p.) if the probability of the $n$-th event is at least $1-O\left(1 / n^{c}\right)$ for some $c \geq 1$. Different sector orientations are specified by an angle that gives the deviation from 


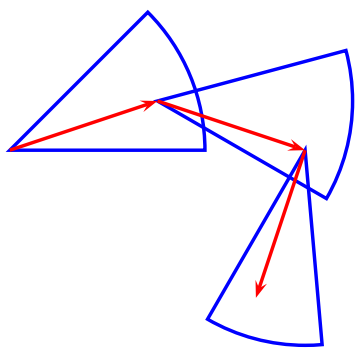

Figure 1: Mote connection through the scanning area

the horizontal axis. All our angle calculations are made in arithmetic modulo $2 \pi$.

\section{The model}

We assume that the terrain to be covered is an square area of size $D \times D$. An appropriate scaling can map it to the unit square $[0,1]^{2}$. We also assume that an angle $\alpha$ is fixed.

A mote can orient its laser beam in any position of a prescribed scanning area of $\alpha$ radians, however the mote can receive light from any point, within distance $r$, which is "looking" to it. This model gives raise to a directed graph model. We define a random digraph, where the vertices are the motes, and given vertices $i$ and $j$, there is an arc $(i, j)$ if $j$ lies in the sector with center $i$ defined by $r$ and $\alpha$ (see Figure 1). We formally define the sector graphs and the random sector graphs,

Definition 2.1. A graph is a unit sector graph if each vertex can be mapped to the center of a closed unit radius sector with amplitude $\alpha$, in the plane, in such a way that there is an arc $(u, v)$ if and only if $v$ lies inside the sector associated to $u$.

Given a set $V$ of $n$ points in the unit square $[0,1]^{2}$, a sequence $B=\left(b_{1}, \ldots, b_{n}\right)$ of angles and a distance $r \in[0,1]$, the corresponding scaled sector graph is the graph $\mathcal{G}_{\alpha}(V, B, r)$ where vertex $i$ has as associated sector the portion of the disk with radius $r$ delimited by angles $b_{i}$ and $b_{i}+\alpha$ with respect to the horizontal axis anticlockwise.

Observe that if $\alpha=2 \pi$, any pair of vertices in a scaled sector graph have bidirectional communication, so that the associated undirected graph is a geometric graph, recall than in a geometric random graphs two points $x$ and $y$ are connected whenever $d(x, y) \leq r$.

The random graph model is defined as follows:

Definition 2.2. Let $X=\left(X_{i}\right)_{i \geq 1}$ be a sequence of independently and uniformly distributed (i.u.d.) random points in $[0,1]^{2}$, let $B=\left(b_{i}\right)_{i \geq 1}$ be a sequence of i.u.d angles and let $\left(r_{i}\right)_{i \geq 1}$ be a sequence of numbers in $[0,1]$, For any natural $n$, we write $\chi_{n}=\left\{X_{1}, \ldots, X_{n}\right\}$ and $\beta_{n}=\left\{b_{1}, \ldots, b_{n}\right\}$. We call $\mathcal{G}_{\alpha}\left(\chi_{n}, \beta_{n}, r_{n}\right)$ the random scaled sector graph with $n$ nodes.

Figure 2 shows two random sector graphs, one with $\alpha=\frac{4}{9} \pi$ and the other with $\alpha=2 \pi$.

The objective of the network is monitoring the terrain. To do so, we assume that the terrain is dissected in a grid of $s$ cells, each of size $s^{-1 / 2} \times s^{-1 / 2}$. This grid serves as reference 

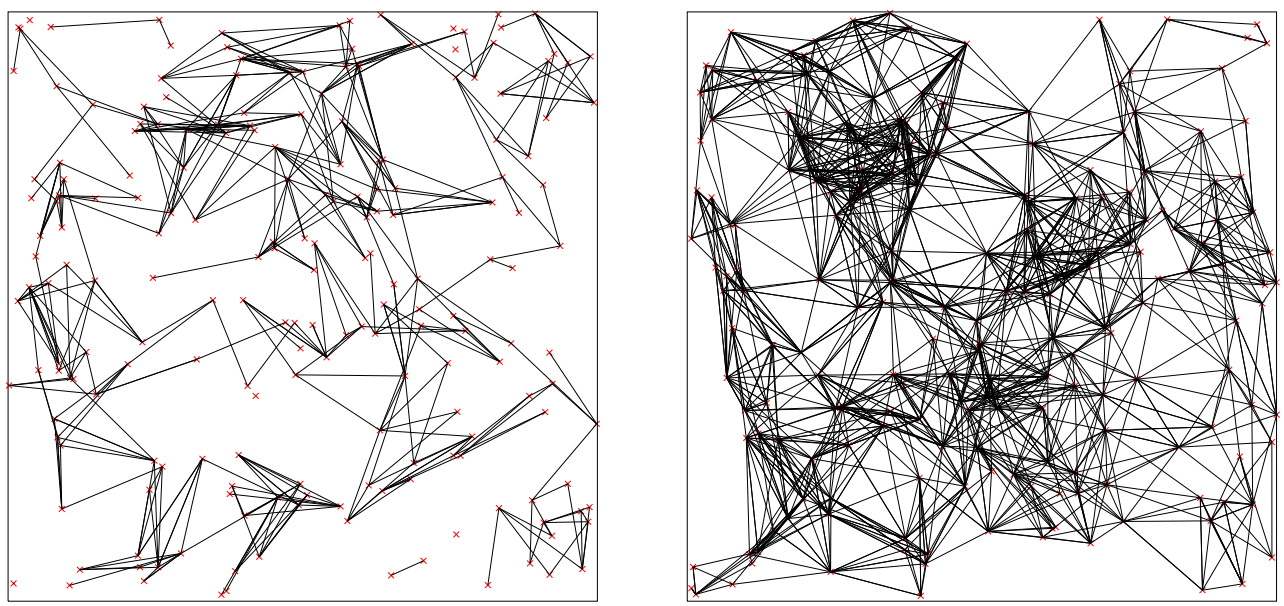

Figure 2: Two random sector graphs with $\alpha=\frac{4}{9} \pi$ and $\alpha=2 \pi$ for $n=200$ and $r=0.2$

position for the motes, and the data reported from a mote refers to the cell in the grid that contains it. The parameter $s$ is selected to represent the sensing precision of the network. Observe that the parameter $s$ controls the scalability of the network in two natural ways: a desired increment of precision or a desire to cover a bigger area results in an increase of $s$.

In the process of scattering the motes, some of them may break down or fall upside down and become inoperative, some may be hidden from the BTS and for some pair of motes the line of sight may be blocked. We assume that motes fall uniformly at random in the terrain and that each mote can fail, independently at random, with probability $f_{o}$. Operative motes may not communicate with the BTS with probability $f_{b}$. Also, we assume that $f_{c}$ is the probability that the line of sight allowing communication from one operative mote to another operative mote is interrupted by some obstacle.

We use the following parameters for a smart dust network: $n$ is the number of motes; $r$ is the laser range of the motes; $\alpha$ is the laser scanning angle; $s$ is the number of cells in the grid; $p_{o}$ is the probability that a mote is operative $\left(f_{o}=1-p_{o}\right) ; p_{b}$ is the probability that an operative mote can communicate with the BTS $\left(f_{b}=1-p_{b}\right) ; p_{c}$ is the probability that the line of sight between two motes is not interrupted $\left(f_{c}=1-p_{c}\right)$.

Now we introduce the random model used in the paper.

Definition 2.3. Let $X=\left(X_{i}\right)_{i \geq 1}$ be a sequence of i.u.d. random points in $[0,1]^{2}$ and let $B=$ $\left(b_{i}\right)_{i \geq 1}$ be a sequence of i.u.d angles. The random smart dust network $\mathcal{S D}_{n}\left(X, B, \alpha, r, s, p_{o}, p_{b}, p_{c}\right)$ is a network of $n$ motes $X_{1}, \ldots, X_{n}$ where $X_{i}$ is operative with probability $p_{o}, X_{i}$ can communicate with the BTS with probability $p_{b}$, and $X_{i}$ and $X_{j}$ can communicate with probability $p_{c}$ if $X_{j}$ is contained in the sector of radius $r$ and angle $\alpha$ starting at $\beta_{i}$.

The system just defined is a subgraph of a random scaled sector graph: each vertex is removed with probability $f_{o}$ and each arc is removed with probability $f_{c}$. Furthermore each vertex gets label 1 with probability $p_{b}$ or label 0 with probability $f_{b}$. We will refer to the motes with label 1 as the communicating motes. 


\section{Network parameters}

Our first result determines the relationship between the number of motes and the sensing precision. Notice that, in order to perform the monitoring task, each sensing cell should contain at least one operative mote. We use a variation of the balls and bins problem; its proof is an adaptation of the classical proof of the coupon collector problem [MR95]:

Lemma 3.1. Assume that balls are successively thrown at random to $n$ bins. The bins are placed behind a wall, with probability $p$ a ball will fail to cross the wall, however if it succeeds the ball has equal probability to fall in any of the bins.

Let $X$ be the random variable denoting the number of trials for having at least one ball in each bin. Then, for any $c \in \mathbb{R}$,

$$
\lim _{n \rightarrow \infty} \operatorname{Pr}[X \leq(n \ln n-c n) / p]=e^{-e^{c}} \text { and } \lim _{n \rightarrow \infty} \operatorname{Pr}[X \geq(n \ln n+c n) / p]=1-e^{-e^{-c}} .
$$

Taking into account that motes correspond to balls and cells to bins, and that as the value $c$ is increased, the probabilities rapidly approach 1 , we get the following result:

Theorem 3.1. Let $\epsilon>0$. Let $\mathcal{S D}_{f(s)}\left(X, B, \alpha, r, s, p_{o}, p_{b}, p_{c}\right)$ be a random smart dust system with $f(s)$ motes. Then, if $f(s) \geq(1+\epsilon)(s \ln s) / p_{o}$, with probability tending to one as $s$ tends to infinity, each of the $s$ cells in the grid contains at least one operative mote. Also, if $f(s) \leq(1-\epsilon)(s \ln s) / p_{o}$, with probability tending to one as $s$ tends to infinity, some of the $s$ cells will not contain any operative mote.

In other words, the previous results says that, with high probability, a random smart dust system with $n$ motes can monitor $s=O(n / \ln n)$ cells, when $p_{o}$ is a constant.

In the following, to diminish boundary effects, we focus our attention to interior motes, that is, motes whose distance from the boundaries of $[0,1]^{2}$ is greater than $r$. Given a smart dust system $D=\mathcal{S D}_{n}\left(X, B, \alpha, r, s, p_{o}, p_{b}, p_{c}\right)$, denote by $I(D)$ the set of interior operative motes of $D$. Let $\delta_{\text {in }}(D), \delta_{\text {out }}(D), \Delta_{\text {in }}(D)$ and $\Delta_{\text {out }}(D)$ denote respectively the minimal indegree, the minimal out-degree, the maximal in-degree, and the maximal out-degree over all motes in $I(D)$. In practice this means we have to embed the area to be monitored, into non-overlapping square grids large enough to cover the whole area.

Given the laser range $r$ and the number of cells $s$, the value $g=r / \sqrt{s}$ is another relevant parameter that will appear in the analysis of random smart dust systems. We will assume that $g$ is a positive constant.

In the remaining of the paper we will use the term $\epsilon$-normalized random smart dust system to refer to a random smart dust system, $D_{n}=\mathcal{S D}_{n}\left(X, B, \alpha, r, s, p_{o}, p_{b}, p_{c}\right)$, with $n=(1+\epsilon)(s \ln s) / p_{o}$ motes and such that $g=r / \sqrt{s}$ is a constant and $\epsilon \geq \epsilon_{0}$. Here, $\epsilon_{0}$ is a constant that only depends on $g$.

Our first result gives basic parameters of the subjacent random graph related to connectivity and degree.

Given a $\epsilon$-normalized smart dust system $\mathcal{S D}_{n}\left(X, B, \alpha, r, s, p_{o}, p_{b}, p_{c}\right)$, we dissect the unit square into $(\gamma r)^{-2}$ boxes, each of size $\gamma r \times \gamma r$ with

$$
\gamma=\left(\left\lfloor\frac{2}{\epsilon r}\right\rfloor r\right)^{-1} .
$$


Observe that $1 /(\gamma r)=\left\lfloor\frac{2}{\epsilon r}\right\rfloor$ is an integer and that $\gamma \rightarrow \epsilon / 2$ and that that $\epsilon / 4 \leq \gamma \leq \epsilon$ for $s$ sufficiently large. We call such a dissection the fine dissection. Our first results gives bounds for the number of points inside a box.

Lemma 3.2. In a $\epsilon$-normalized smart dust system $\mathcal{S D}_{n}\left(X, B, \alpha, r, s, p_{o}, p_{b}, p_{c}\right)$, every box of the fine dissection contains w.h.p. at most $(1+\gamma)(1+\epsilon) g^{2} \ln s$ points and at least $(1-\gamma)(1+$ $\epsilon) g^{2} \ln s$ points, where $g=r / \sqrt{s}$.

Proof. Choose a box in the dissection and let $Y$ be the random variable counting the number of points of $\chi_{n}$ in this box. As the points in $\chi_{n}$ are i.u.d. and the probability that a mote is operative is $p_{o}$,

$$
\mu=\mathbf{E}[Y]=(\gamma r)^{2} p_{o} n=\frac{\gamma^{2}}{s} p_{o}(1+\epsilon) \frac{s \ln s}{p_{o}}=(1+\epsilon) g^{2} \ln s .
$$

Using Chernoff bounds,

$$
\operatorname{Pr}[Y \geq(1+\gamma) \mu] \leq e^{-\gamma^{2} \frac{\mu}{3}} \leq e^{-\frac{\gamma^{2}(1+\epsilon) g^{2}}{3} \ln s}
$$

and

$$
\operatorname{Pr}[Y \leq(1-\gamma) \mu] \leq e^{\gamma^{2} \frac{\mu}{3}} \leq e^{-\frac{\gamma^{2}(1+\epsilon) g^{2}}{3} \ln s} .
$$

The number of boxes is $(\gamma r)^{-2}=\frac{s}{\gamma^{2} g^{2}}$ so by Boole's inequality, the probability that for some box the number of points in the box is less than $(1-\gamma)(1+\epsilon) g^{2} \ln s$ or more than $(1+\gamma)(1+\epsilon) g^{2} \ln s$ is bounded by $2 \frac{s}{\gamma^{2} g^{2}} e^{-\frac{\gamma^{2}(1+\epsilon) g^{2}}{3} \ln s}$, which can be made $O\left(s^{-1}\right)$ by selecting an adequate $\epsilon$.

Our next result gives sharp estimations of the expected maximum and minimum of the in and out degree of interior vertices. Observe that in a random scaled sector graph, given two vertices $x$ and $y$, within distance $r_{n}$, the pair $(x, y)$ is an edge if $y$ is inside the sector with radius $r_{n}$ and $\alpha$ degrees. This property holds with probability $\frac{\alpha}{2 \pi}$.

A sector spans over several boxes of the fine dissection. Consider a sector, the boxes that are completely in the sector are called the entire boxes and the boxes that are inside or cut by the sector are called the touched boxes.

In order to simplify notation, we define two auxiliary constants:

$$
\kappa_{1}=\kappa_{1}(\alpha)=(1-\gamma) \frac{\alpha-4 \alpha \gamma-8}{2} \quad \text { and } \quad \kappa_{2}=\kappa_{2}(\alpha)=(1+\gamma) \frac{\alpha+4 \alpha \gamma+8}{2} .
$$

One of the restriction for the selected $\epsilon$ in the normalized smart dust systems is that $\kappa_{1}(\alpha)>1$.

Theorem 3.2. Let $G_{n}=\mathcal{S D}_{n}\left(X, B, \alpha, r, s, p_{o}, p_{b}, p_{c}\right)$ be a $\epsilon$-normalized smart dust system. Then, w.h.p.

$$
(1-\gamma) \kappa_{1}(1+\epsilon) p_{c} g^{2} \ln s \leq \delta_{\text {in }}\left(G_{n}\right) \leq \Delta_{\text {in }}\left(G_{n}\right) \leq(1+\gamma) \kappa_{2}(1+\epsilon) p_{c} g^{2} \ln s,
$$

and

$$
\kappa_{1}(1+\epsilon) p_{c} g^{2} \ln s \leq \delta_{\text {out }}\left(G_{n}\right) \leq \Delta_{\text {out }}\left(G_{n}\right) \leq \kappa_{2}(1+\epsilon) p_{c} g^{2} \ln s
$$


Proof. Given any vertex $u$ in $G_{n}$, let $S_{u}$ denote the sector associated to $u$. Let us bound the maximum number of boxes in the fine dissection, intersected by the perimeter of $S_{u}$. Notice that any segment with length $r_{n}$ cuts at most $\frac{2}{\gamma}$ cells and any arc with radius $r_{n}$ and angle $\alpha$ cuts at most $\frac{2 \alpha}{\gamma}$ cells. Therefore, as the area of $S_{u}$ is $\frac{\alpha}{2} r_{n}^{2}$, the total number of entire boxes is at least $\frac{\alpha}{2 \gamma^{2}}-\frac{4+2 \alpha}{\gamma}$. Moreover, the number of touched boxes is at most $\frac{\alpha}{2 \gamma^{2}}+\frac{4+2 \alpha}{\gamma}$.

Let $v$ be a vertex and $C_{v}$ the disk with center $v$ and radius $r_{n}$. The indegree of $v$ is the number of vertices inside $C_{v}$, which look to $v$. The number of entire boxes of the fine dissection is at least $\frac{\pi}{\gamma^{2}}-\frac{4 \pi}{\gamma}$, and the number of touching boxes is at most $\frac{\pi}{\gamma^{2}}+\frac{4 \pi}{\gamma}$. Therefore, using lemma 3.2, w.h.p., the number $x_{v}$ of operative motes in $C_{v}$ that can communicate with $v$ is at least

$$
(1-\gamma)(1+\epsilon) p_{c} g^{2} \ln s\left(\frac{\pi}{\gamma^{2}}-\frac{4 \pi}{\gamma}\right)=(1-\gamma) \kappa_{1}(1+\epsilon) p_{c} g^{2} \ln s
$$

and at most

$$
(1+\gamma)(1+\epsilon) p_{c} g^{2} \ln s\left(\frac{\pi}{\gamma^{2}}+\frac{4 \pi}{\gamma}\right)=(1+\gamma) \kappa_{1}(1+\epsilon) p_{c} g^{2} \ln s
$$

As the probability that a vertex $u$ in the circle looks at $v$ is $\alpha / 2 \pi$, then $\mathbf{E}\left[d_{\text {in }}(v)\right]=\frac{\alpha}{2 \pi} p_{c} x_{v}$. Using Chernoff's bounds and Boole's inequality, we get

$$
\operatorname{Pr}\left[\delta_{\text {in }}\left(G_{n}\right) \leq(1-\gamma) \kappa_{1}(1+\epsilon) p_{c} g^{2} \ln s\right] \leq n e^{-\frac{\gamma^{2}}{2} \frac{\alpha}{2 \pi} \kappa_{1}(1+\epsilon) p_{c} g^{2} \ln s},
$$

and

$$
\operatorname{Pr}\left[\Delta_{\text {in }}\left(G_{n}\right) \geq(1+\gamma) \kappa_{2}(1+\epsilon) p_{c} g^{2} \ln s\right] \leq n e^{-\frac{\gamma^{2}}{3} \frac{\alpha}{2 \pi} \kappa_{1}(1+\epsilon) p_{c} g^{2} \ln s} .
$$

And the probability of any one of these two events is $O\left(s^{-1}\right)$ by selecting an adequate $\epsilon$.

Next we will show that normalized smart dust systems are almost strongly connected w.h.p. That is, there is a directed path joining any pair of interior points. We use the following result on random geometric graphs.

Theorem 3.3 ([Pen99]). Random geometric graphs $\mathcal{G}\left(\chi_{n}, r_{n}\right)$ are connected w.h.p., when $r_{n} \geq \sqrt{c \log n / n}$ for some big enough constant $c$.

For a normalized smart dust system we will show the existence (w.h.p.) of an emulating directed path of length at most four, for any of the two orientations of an edge in the corresponding associated geometric supergraph.

Lemma 3.3. Let $G_{n}=\mathcal{S D}_{n}\left(X, B, \alpha, r, s, p_{o}, p_{b}, p_{c}\right)$ be a $\epsilon$-normalized smart dust system. Then, w.h.p., given a pair $(x, y)$ of interior operative motes with $d(x, y) \leq r$, there is a directed path from $x$ to $y$ in $G_{n}$ of length at most 4 .

Proof. If $(x, y) \in E\left(G_{n}\right)$, then we are done. Otherwise, we will show that, w.h.p., there is a directed path from $x$ to $y$ in $E\left(G_{n}\right)$.

Let $C_{x}$ be the circumference with center $x$ and radius $r$, notice that $y$ is inside $C_{x}$. Let $S_{x}$ be the sector associated to $x$, and let $z_{1}$ be the closest point in $S_{x} \cap C_{x}$ to the intersection of radius containing $y$ with $C_{x}$. Let $C_{1}$ be the circumference with radius $r_{n}$ and center $z_{1}$ and 


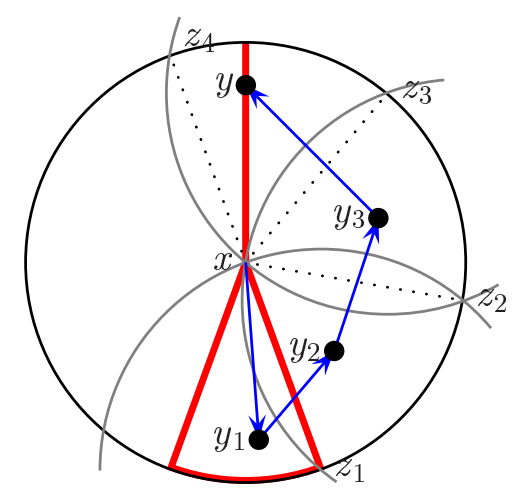

Figure 3: An emulating path

$z_{2}$ be the intersection of $C_{1}$ and $C_{x}$. Let $C_{2}$ be the circumference with radius $r_{n}$ and center $z_{2}$ and $z_{3}$ be the intersection of $C_{2}$ and $C_{x}$. Finally, let $C_{3}$ be the circumference with radius $r_{n}$ and center $z_{3}$ and let $z_{4}$ be the intersection of $C_{3}$ and $C_{x}$ (see Figure 3 ).

Observe that all the points in $S_{x}$ (or a portion of $\pi / 3$ of it) are within distance $r$ of the sector defined by $z_{1}$ and $z_{2}$. The points in this sector are within distance $r$ of the sector defined by $z_{2}$ and $z_{3}$. The points in this last sector are within distance $r$ of the sector defined by $z_{3}$ and $z_{4}$. Furthermore $y$ must be inside one of the four sectors.

Assume without loose of generality that $x$ and $y$ are in the worst possible case, when the four sectors are needed. We will show the existence (w.h.p.) of three operative motes $y_{1}, y_{2}, y_{3}$, one from each sector, so that the four arcs $\left(x, y_{1}\right),\left(y_{1}, y_{2}\right),\left(y_{2}, y_{3}\right),\left(y_{3}, y\right)$ exist in $G_{n}$.

We can bound the probability that such a selection of connected vertices exists, taking into account that, in a normalized smart dust system, w.h.p, such sector has $\approx c_{1}(1+\epsilon) g^{2} \ln s$ points for some constant $c_{1}$ and, the probability of having the arc $(x, y)$ is $\frac{\alpha}{2 \pi} p_{c}$. Such a path does not exists with probability at most

$$
\left(\left(1-\frac{\alpha}{2 \pi} p_{c}\right)^{c_{1}(1+\epsilon) g^{2} \ln s}\right)^{4}
$$

Combining the previous result with Theorem 3.3 we have th efollowing result.

Theorem 3.4. Let $G_{n}=\mathcal{S D}_{n}\left(X, B, \alpha, r, s, p_{o}, p_{b}, p_{c}\right)$ be a $\epsilon$-normalized smart dust system. Then, w.h.p., given a pair $(x, y)$ of interior operative motes, there is a directed path from $x$ to $y$ in $G_{n}$.

Proof. By Theorem 3.3 we know that w.h.p., the graph $\mathcal{G}\left(\chi_{n}, r_{n}\right)$ is connected. Using Boole's inequality and Lemma 3.3, the probability of an edge $\{x, y\}$ in $\mathcal{G}\left(\chi_{n}, r_{n}\right)$ failing to be emulated by a path in $\mathcal{G}_{\alpha}\left(\chi_{n}, \beta_{n}, r_{n}\right)$, with length at most 4 (in one of the two possible orientations) is $O\left(n^{-c}\right)$.

Putting together Theorems 3.2 and 3.4 we have:

Theorem 3.5. Let $D_{n}=\mathcal{S D}_{n}\left(X, B, \alpha, r, s, p_{o}, p_{b}, p_{c}\right)$ be a $\epsilon$-normalized random smart dust system. Let $\gamma>0$ and define $\kappa_{1}=(1-\gamma)(\alpha-4 \alpha \gamma-8) / 2$ and $\kappa_{2}=(1+\gamma)(\alpha+4 \alpha \gamma+8) / 2$. Then, as $s$ grows, the probability of the following events rapidly approaches 1 : 
- For all $x, y \in I\left(D_{n}\right)$, there is a directed path from $x$ to $y$ in $D_{n}$.

- $(1-\gamma) \kappa_{1}(1+\epsilon) p_{c} g^{2} \ln s \leq \delta_{\text {in }}\left(D_{n}\right) \leq \Delta_{\text {in }}\left(D_{n}\right) \leq(1+\gamma) \kappa_{2}(1+\epsilon) p_{c} g^{2} \ln s$.

- $\kappa_{1}(1+\epsilon) p_{c} g^{2} \ln s \leq \delta_{\text {out }}\left(D_{n}\right) \leq \Delta_{\text {out }}\left(D_{n}\right) \leq \kappa_{2}(1+\epsilon) p_{c} g^{2} \ln s$.

\section{The localization algorithm}

Once deployed, the first task for a smart dust network is to find the position of each operative interior mote. Motes that can communicate with the BTS will receive its coordinates from th BTS (it suffices to include the emission angle in the BTS message [KKRP99]). The remaining motes will have to compute their coordinates based on the coordinates of other motes and the angle of incidence of the incoming laser beams. In this section, we present and analyze a localization algorithm for that purpose. For the moment we assume that the laser beams can not produce interference and distorsion of information.

Recall the following result from geometry.

Lemma 4.1. Let $A, B, C$ and $O$ be three points in the plane such that $O$ does not lie in the circumference described by $A, B$ and $C$. Then, the coordinates of $O$ can be computed using the coordinates of $A, B$ and $C$ and the angles $\widehat{A O B}$ and $\widehat{A O C}$.

As the probability that four random points lie in a circumference is negligible, a mote can compute its position if it can receive the coordinates of three other motes. Therefore, the localization problem is captured by the following general graph theoretic problem:

Input: A graph $G=(V, E)$, a subset of vertices $S \subset V$, and two integers positive integers $k$ and $d$.

Question: Is it possible to obtain a partition of $V$ into $k$ layers $S_{0}, S_{1}, \ldots, S_{k}$ such that $S=S_{0}$ and such that for any $i>0$ and any vertex $v \in S_{i}, v$ has at least $d$ neighbors in the previous $i$ levels?

In the case of an undirected graph and $k=d=1$, the above problem is equivalent to test if $S$ is a dominating set. As $S$ is given, for other values of $k$ and $d$ the problem can be solved in polynomial time using a greedy procedure. Once we have the set $S_{i-1}$, we construct $S_{i}$ by placing into it all the accessible vertices that satisfy the requirements.

Inspired in this greedy solution, we propose the algorithm localize given in Figure 4, to solve the localization stage for smart dust systems.

Observe that any mote is required to enter in at most three awake-compute-sleep activations and to perform a full scan. Therefore, the energy consumption of this algorithm is very low. It remains to consider the runtime of this algorithm and whether it will leave motes without knowing their coordinates.

Theorem 4.1. Let $D_{n}=\mathcal{S D}_{n}\left(X, B, \alpha, r, s, p_{o}, p_{b}, p_{c}\right)$ be a $\epsilon$-normalized random smart dust system. Then, as $s$ grows, the probability that all operative interior motes know their position, after a constant number of steps, rapidly approaches 1 .

Proof. Let $Z$ be a random variable that counts the number of successes of Bernoulli trials with probability of success $p$, and let $\mu=\mathbf{E}[Z]$. Using Chernoff bounds

$$
\operatorname{Pr}[Z \leq 3] \leq e^{-\left(\frac{\mu}{3}-2\right)} .
$$


- Phase 0

- The BTS performs with its laser a full scanning of the terrain, sending the corresponding positions.

- Motes that can receive the BTS signal take note of their coordinates.

- Phase $i,(i \geq 1)$

- Every mote that knows its coordinate performs a scanning along all its angle sending its coordinate.

- Every mote that does not know its coordinate waits to receive three positions, computes its coordinates, and performs a scanning along all its angle sending its coordinate.

Figure 4: The localize protocol

Therefore in the case that $\mu=c \ln n$

$$
\operatorname{Pr}[Z \geq 3] \geq 1-\frac{e^{2}}{n^{-\frac{c}{3}}}
$$

This is the case when $Z_{u}$ counts the number of motes $v$ that know their coordinates, such that the link $(u, v)$ exits. In this case $\mu \sim \kappa_{1}(1+\epsilon) p_{c} g^{2} \ln s$. Therefore, with probability $1-s^{-\frac{(1+\epsilon) p_{c} g^{2}}{3}}$, a mote will acquire its coordinates after one iteration. Provided $p_{b}=\Omega\left(\frac{1}{\ln s}\right)$, an application of Chernoff's bounds show that a constant fraction of motes will acquire its coordinates in one phase w.h.p.

Iterating the argument, let $Z_{u}^{i}$ count the number of motes $v$ that know their coordinates during the $i$-th phase, such that the link $(u, v)$ exists, for those $u$ that have not acquired coordinates at the $i$-th step. The probability of failing is small, so after a constant number of steps the probability of failing is $s^{-2}$.

Let $t_{1}$ be the time needed by the BTS to send a signal to the whole grid, and let $t_{2}$ be the time need by a mote to perform a full scan of $\alpha$ degrees.

Theorem 4.2. There exists a constant $\tau$, such that, for any $\epsilon$-normalized random smart dust system $D_{n}=\mathcal{S D}_{n}\left(X, B, \alpha, r, s, p_{o}, p_{b}, p_{c}\right)$. The probability that after $t_{1}+\tau t_{2}$ units of time, all operative interior motes know their position, rapidly approaches 1 , as $s$ grows.

Table 1 shows the empirical results obtained using the localization protocol in Figure 4. From these values, one can observe that the localization phase will succeed very quickly (five phases at most) when $p_{c}$ is low, but that for a low number of motes and a high value of $p_{c}$ the algorithms fails to assign coordinates to each interior operative mote. Moreover, in those cases, more than $90 \%$ of the interior motes are able to compute their position.

\section{Synchronization issues}

As the communication is optical, receiving simultaneous messages is undesirable. It is possible to avoid interferences by timing the scanning and synchronizing the internal clock of all the motes. 


\begin{tabular}{rrrrrr}
\multicolumn{6}{c}{$p_{o}=p_{b}=0.5, \alpha=40^{\circ}$} \\
\hline & \multicolumn{5}{c}{$p_{c}$} \\
\cline { 2 - 6 }$n$ & 0.0 & 0.1 & 0.2 & 0.3 & 0.4 \\
\hline 1000 & 2 & 5 & - & - & - \\
2000 & 2 & 2 & - & - & - \\
3000 & 2 & 2 & 3 & 3 & 4 \\
4000 & 2 & 2 & 2 & 4 & - \\
5000 & 2 & 2 & 2 & - & 5 \\
6000 & 1 & 2 & 3 & 4 & 4 \\
7000 & 2 & 2 & - & 3 & 4 \\
8000 & 2 & 2 & 3 & 3 & - \\
9000 & 2 & 2 & 2 & 3 & 3 \\
10000 & 2 & 1 & 2 & 2 & 4 \\
11000 & 2 & 2 & 2 & 4 & 4 \\
12000 & 2 & 2 & 2 & 4 & 3 \\
13000 & 2 & 2 & 2 & 3 & 3 \\
14000 & 2 & 2 & 3 & 2 & 3 \\
15000 & 2 & 2 & 2 & 4 & 3 \\
\hline
\end{tabular}

\begin{tabular}{rrrrrr}
\multicolumn{5}{c}{$p_{o}=0.25, p_{b}=0.5, \alpha=40^{\circ}$} \\
\hline & \multicolumn{5}{c}{$p_{c}$} \\
\cline { 2 - 6 }$n$ & 0.0 & 0.1 & 0.2 & 0.3 & 0.4 \\
\hline 1000 & 3 & 3 & 5 & - & - \\
2000 & 2 & 2 & 5 & - & - \\
3000 & 2 & 3 & 4 & - & - \\
4000 & 3 & 2 & - & 4 & - \\
5000 & 3 & 2 & 4 & - & - \\
6000 & 2 & 4 & 4 & 3 & - \\
7000 & 2 & 3 & 3 & 3 & - \\
8000 & 2 & 2 & 2 & 3 & 5 \\
9000 & 2 & 2 & 3 & 3 & - \\
10000 & 2 & 2 & 3 & 3 & - \\
11000 & 2 & 2 & 3 & 4 & - \\
12000 & 2 & 2 & 2 & 3 & - \\
13000 & 2 & 2 & 2 & 4 & 5 \\
14000 & 2 & 2 & 3 & 4 & 4 \\
15000 & 2 & 2 & 3 & 4 & 4 \\
\hline
\end{tabular}

Table 1: Number of phases necessary to receive coordinates depending on $n, p_{b}$ and $p_{c}$. Dashes mean failure to assign the coordinate to each interior motes.

We assume that the motes have a precomputed estimation of the time needed to perform a complete scanning of $2 \pi$. They can agree on a period of time to perform a synchronized scanning in which, at each time instant, all active beams are oriented in the same direction. Furthermore they have to identify the angle formed by its scanning zone with a reference axis.

All this synchronization steps can be solved during the execution of the localization phase. To do so, motes will encode in their messages the following information:

- The current time.

- Its coordinates.

- The level at which the coordinates were obtained.

- The total number of phases to be executed.

Observe that the time and the number of phases will be provided by the BTS and the information should be broadcasted to all the remaining motes.

Once a mote gets a message, it can synchronize its clock and compute an estimation of the time to finish the protocol. Upon receiving three communications, a mote can compute its phase, by adding one to the phase of the last communicating mote. The angle of the steering beam can be obtained using the triangulation.

When the mote sends a message, it will start at the time assigned to its angle, thus acting synchronously. The only interference that can arise in this scheme is due to the fact of having three aligned motes with coincident lines of sight. But this event happens with negligible probability. 
- Phase 0:

- The source motes get level number 0. They perform a full scan, sending the message and level number 0. After that they go into an sleeping phase.

- An awakened mote gets level 1.

- Phase $i,(i \geq 1)$

- All motes with level $i$ perform a full scan, sending the message and its level number incremented by one. After that they enter in a sleeping phase.

- Any mote that is awakened for the first time, gets level number $i$.

Figure 5: The simple-bro protocol

\section{Broadcasting from BTs to mote}

Another of the issues to be considered is the broadcasting of messages originated in the BTS. We propose the protocol simple-bro given in Figure 5, which is a classical flooding algorithm, with multiple source points.

First we analyze the proposed broadcasting protocol in the random geometric graph defined by the operative motes. Recall that the random sources correspond to the communicating motes.

Theorem 6.1. Let $D_{n}=\mathcal{S D}_{n}\left(X, B, \alpha, r, s, p_{o}, p_{b}, p_{c}\right)$ be an $\epsilon$-normalized smart dust and let $G_{n}$ be the geometric graph defined by the operative motes and $r$. Then, w.h.p., after performing $\left\lceil\sqrt{\frac{2 p_{o}}{p_{b}(1+\epsilon) g^{2}}}\right\rceil$ phases of the simple-bro protocol, all vertices in $G_{n}$ know the message originated in the random sources.

Proof. Let's dissect the unit square into $p_{b} \frac{(1+\epsilon) s}{p_{o}}$ boxes. Each box is a square with dimension $\sqrt{\frac{p_{o}}{p_{b}(1+\epsilon) s}}$. Using the coupon collector result, any square in this dissection will get w.h.p. a source provided the number of sources is roughly the number of boxes times its logarithm. But,

$$
\frac{p_{o}}{p_{b}(1+\epsilon) s} \ln \left(\frac{p_{o}}{p_{b}(1+\epsilon) s}\right) \leq(1+\delta) \frac{p_{o}}{p_{b}(1+\epsilon) s} \ln s
$$

Which is the expected number of communicating motes.

We further dissect each square with dimension $\sqrt{\frac{p_{o}}{p_{b}(1+\epsilon) s}}$ into identical squares with dimension $\frac{r}{\sqrt{2}}$. Thus, the number of small boxes that fit in one dimension is

$$
\sqrt{\frac{p_{o}}{p_{b}(1+\epsilon) s}} \frac{\sqrt{2}}{r_{n}}=\sqrt{\frac{2 p_{o}}{p_{b}(1+\epsilon) g^{2}}} .
$$

Notice that all the vertices in a small box are interconnected among them. Moreover, any vertex in the box is also connected to some vertices in neighboring boxes, except the corner points that will not reach the diagonal neighboring box. Therefore, the sources will cover in one step its small boxes, and some vertex in all but at least one neighboring small boxes. Continuing the flooding, after performing $\left\lceil\sqrt{\frac{2 p_{o}}{p_{b}(1+\epsilon) g^{2} a_{n}}}\right\rceil$ phases, all the points in the big 
box, will collect the message. As the sources can initiate the broadcasting independently, we attain the desired result.

The combination of the above result with Lemma 3.3 yields the following result.

Theorem 6.2. Let $D_{n}=\mathcal{S D}_{n}\left(X, B, \alpha, r, s, p_{o}, p_{b}, p_{c}\right)$ be a $\epsilon$-normalized random smart dust system. Then, as $s$ grows, with probability rapidly approaching 1 , after performing $4\left\lceil\sqrt{\frac{2 p_{o}}{p_{b}(1+\epsilon) g^{2}}}\right\rceil$ phases of the simple-bro protocol, a message originated in the BTS will be broadcasted to all interior motes.

The energy consumed by a mote in the proposed protocol is bounded to a maximum of a complete scanning per mote. As described in Section 5, the scanning can be synchronized to eliminate interference.

\section{Route establishment from mote to BTS}

Before exploiting the network of sensors, a smart dust system must establish a routing so that any operative interior mote can send its information to the BTS. Motes that can communicate directly with the BTS will simply transmit passively their information when the BTS queries them. The remaining motes will have to send their information actively by the way of multiple hops. Since the communication between motes is not bidirectional, it is not allowed to simply reverse the paths found by the algorithm in Figure 5 .

To establish a route from the mote to the BTS, we propose the protocol simple-link given in Figure 6. Notice that the computed set of routes from motes to BTS form an oriented forest with roots in the communicating motes. The energy consumed by this protocol is bounded to a maximum of a complete scanning per phase and a laser positioning. As in Section 5 , the scanning can be organized to avoid interference.

Theorem 7.1. Let $D_{n}=\mathcal{S D}_{n}\left(X, B, \alpha, r, s, p_{o}, p_{b}, p_{c}\right)$ be a $\epsilon$-normalized random smart dust system. Then, as $s$ grows, with probability rapidly approaching 1 , after performing $4 /\left\lceil\sqrt{\left(p_{o} p_{b} g\right)}\right\rceil$ phases of the simple-gather protocol, a message originated in the BTS will be broadcasted to all interior motes.

Proof. Using Theorem 6.2, for any randomly selected set of communicating nodes, we have that the broadcasting time is at most $4\left[\sqrt{\frac{2 p_{o}}{p_{b}(1+\epsilon) g^{2}}}\right]$. The simple-trans protocol attempts to replacing the arcs in the broadcasting tree by paths that emulate the reversed direction.

\section{Memory considerations}

Once the routing forest is established, the information from the motes is sent to the BTS. In the proposed schema, the highest need of memory arises in the size of the messages that a mote has to buffer, before sending it to the next mote. Observe that the size of the message increases as the mote gets closer to the BTS, as it contains all the smaller received messages.

The worst case for memory usage, arises when a mote with direct communication with the BTS is responsible for the communication of all the motes inside a disk of radius $h r$, where $h$ is the maximum number of hops needed in the protocol. Thus the mote is the root of a tree. The tree includes at level $i$ all the motes inside a disk of radius $i r$ and outside a disk 
- Phase 0:

- All motes that are not communicating motes perform a full scan, sending its id number.

- Any mote $x$ composes a message $m_{x}$ with all the received id's and keeps it in its memory.

- The communicating motes send $\left\langle x, m_{x}\right\rangle$ to the master.

- The master identifies the id of all the received motes and split them so that any mote that can communicate in one hop gets an assigned communicating mote, in fact the angle to orient the laser to communicate with it.

- This assignation is broadcasted through 4 phases of the simple-bro protocol, so that the motes that have been assigned a node can fix their laser beam in the adequate direction.

- Phase $i,(i \geq 1)$

- All motes that have just fixed its laser direction, send a message $\left\langle x, m_{x}\right\rangle$ in their assigned direction.

- All motes $x$ that do have an assigned direction, compose a message with all the information received and send it.

- The master identifies the id of all the received motes and split them so that any mote that can communicate in $i+1$ hops gets an assigned communicating mote.

- This assignation is broadcasted through $4(i+1)$ phases of the simple-bro protocol, so that the motes that have been assigned to a node can fix their laser beam in the adequate direction.

Figure 6: The simple-link protocol.

of radius $(i-1) r$, with center in the communicating mote. Then, with probability rapidly approaching 1 , the maximum number of single messages arriving at the same time to the communicating mote is of the order $\left(p_{b} p_{o} g\right)^{-3 / 2}$, with. Therefore, we have an upper bound to the length of the longest message.

In the case that the scanning time is large, for example when the BTS is in a plane, fragmentation of information will be undesirable. In those cases, one solution could be to increase the number of motes that communicate with the BTS, by scattering a larger amount of motes (after all, motes are cheap). This alternative will result in a reduction of the number of hops, and therefore, in the reduction of the maximum message's size.

In the situations in which the frequency of scanning is higher, for example a powerful laser in a nearby elevation, the maximum length of the message can be reduced by splitting the motes into prestablished clusters. In the dust system under consideration, all the motes can acquire their coordinates, so a fixed cluster pattern can be used. Assuming the worst case, and taking into account the fact that $(2 k+1) \pi r^{2}$ is the surface of the circular corona with radii $k r$ and $(k+1) r$, we can make a subdivision as the one shown in Figure 7 , in which the cluster of motes are subdivided at distances which are a power of two. To begin the communication, the BTS can compute a time period for each cluster. Each mote computes to which cluster belongs, using as information the value of its coordinates. 


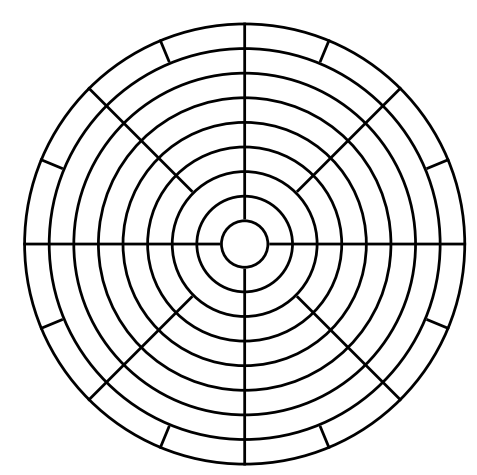

Figure 7: The subdivision scheme for decreasing the memory usage

\section{Conclusions and open problems}

In this paper we put forward a random model for smart dust networks of sensors. Our model, although simple, seems to incorporate the basic technological specification of smart dust systems that communicate through optical links. We have also presented simple protocols that provide a communication layer for smart dust and analyzed its performance. Once this communication layer is established, the system can monitor the sensors and react appropriately. It is worth to notice that the algorithm for the localization phase is distributed and does not require costly centralized computations. This is in contrast to some centralized approaches to solve the localization phase, such as the ones based in semidefinite programming, where the communication is chosen to be radio frequency [Doh00], or the algorithm proposed by [SS01] which requires bi-directional communication.

The proposed static route establishment could also be used to detect and quantify the existence of serious network damage, for example if a fire has destroyed a large part of the network. If some of the motes can communicate with the BTS, running the protocol for a bigger number of phases, all the motes that can communicate with the communicating motes will be reoriented. After a gathering phase the BTS can compute the new map, observing the shape of the damage. This will give a first estimation, however further analysis is needed to design algorithms for detecting small damage in the network and local recovering.

Our model always assumes uniform and independent distributions, and it does not cope with mobility (due to atmospheric conditions motes might fly). In the case that mobility is rare, the network may apply a periodical re-start using the algorithms we have proposed. This may perturb the uniform distribution, so the analysis should be carried under some distribution that deviates from uniform. In the case that mobility is moderate, we believe that a throughly study of a dynamic version of our random model would be of interest. For highly mobile conditions, it seems that laser communication will not be desirable, due to the accuracy needed to orient the laser beam.

Another model for smart dust networks that communicate using radio frequency was considered in [CNS02]. In their setting the network objective is the detection of relevant events that must be forwarded to a wall where the event can be recorded. It will be of interest to analyze the wall problem in our model to be able to compare both models.

We have proposed a new model of geometric graphs to study algorithmic issues arising in the optical communication layer of networks of sensors. The model is of interest by itself 
and might be of use in analyzing and modeling other problems. In particular, it could be easily adapted to medel sensors networks communicating, through radio frequency, with a directional antena. In such a case, interference will play a different and more sigificant role.

Other line that remains open is the study of purely graph theoretical questions on these graphs as, chromatic number, independent set, and others. It is worth noticing that all the problems shown to be NP-complete for unit disk graphs in [CCJ90, DPPS01] can easily be shown NP-complete for unit sector graphs for any fixed angle $\alpha$. Furthermore the results in [BK98] can be adapted to show that the recognition problem is also NP-hard. The results in this paper show that random scaled sector graphs with $n$ vertices and $r_{n}=\sqrt{c \ln n / n}$ are "almost" strongly connected w.h.p. It will be of interest to study the transition phase for connectivity.

We also have obtained tight bounds for the analysis of flooding algorithms in random geometric graphs with vertex faults. To the best of our knowledge, this is the first time these results have been obtained.

\section{References}

[ASSC02] I.F. Akyildiz, W. Su, Y. Sankarasubramanian, and E. Cayirci Wireless sensor networks: a survey. Computer Networks, 38:393-422, 2002.

[AR97a] M. J. Appel and R. P. Russo. The maximum vertex degree of a graph on uniform points in $[0,1]^{d}$. Adv. Applied Probability, 29:567-581, 1997.

[AR97b] M. J. Appel and R. P. Russo. The minimum vertex degree of a graph on uniform points in $[0,1]^{d}$. Adv. Applied Probability., 29:582-594, 1997.

[BK98] H. Breu and D. G. Kirkpatrick. Unit disk graph recognition is NP-hard. Computational Geometry. Theory and Applications, 9(1-2):3-24, 1998.

[CCJ90] B. N. Clark, C. J. Colbourn, and D. S. Johnson. Unit disk graphs. Discrete Mathematics, 86:165-177, 1990.

[CNS02] Ioannis Chatzigiannakis, Sotiris Nikoletseas, and Paul Spirakis. Smart dust local detection and propagation protocols. In ACM Workshop on Principles of Mobile Computing, Toulouse, 2002.

[Doh00] L. Doherty. Algorithms for positioning and data recovery in wireless sensor networks. Master thesis, Dept. of Electrical Engineering and Computer Science, UC Berkeley, 2000.

[DPPS01] J. Díaz, M. D. Penrose, J. Petit, and M. Serna. Approximating layout problems on random geometric graphs. Journal of Algorithms, 39:78-116, 2001.

[DPS98] J. Díaz, J. Petit, and M. J. Serna. Random geometric problems on $[0,1]^{2}$. In J. Rolim, M. Luby, and M. Serna, editors, Randomization and Approximation Techniques in Computer Science, volume 1518 of Lecture Notes in Computer Science, pages 294-306. SpringerVerlag, Berlin, 1998.

[EGHK99] D. Estrin, R. Govindan, J. Heidemann, and S. Kumar. Next century challenges: Scalable coordination in sensor networks. In ACM/IEEE Int. Conf. on Mobile Computing and Networking, pages 263-270, Seattle, 1999.

[KKP00] J. M. Kahn, R. H. Katz, and K. S. J. Pister. Emerging challenges: Mobile networking for smart dust. Journal of Communications and Networks, 2(3):188-196, 2000.

[KKRP99] J.M. Kahn, Katz, R.H., and K.S.J. Pister. Mobile networking for smart dust. In ACM/IEEE Int. Conf. on Mobile Computing and Networking, pages 176-189, Seattle, 1999. 
[MR95] R. Motwani and P. Raghavan. Randomized Algorithms. Cambridge University Press, 1995.

[Pen99] M. D. Penrose. On $k$-connectivity for a geometric random graph. Random Structures \& Algorithms, 15(2):145-164, 1999.

[Pis02] K.S.J. Pister. Personal communication, 2002.

[Sma] Smart Dust Project. http://basics.EECS.Berkeley.EDU/sensorwebs/

[SS01] S.N. Simić and S. Sastry. Distributed localization in wireless ad hoc networks. Manuscript, Dept. of Electrical Engineering and Computer Science, UC Berkeley, 2001. 